\title{
1. Introduction: the impending revolution in video technology, and its impacts on today's and tomorrow's media industries
}

Few questions are fraught with more long-term implications than the way we shape our communications system. If the medium is indeed the message, and if these messages influence people and institutions, then today's technologies and media industries will govern future society, culture, and economy.

It is therefore important to understand that we are on the verge of one of humanity's great leaps in media communications, and consequently also of one of its major disruptions of social and economic arrangements. Based on technological and economic trends, change in media will keep speeding up, and help to generate an unprecedented acceleration in the transformation of culture and politics.

These are strong words, but we will substantiate them in this book, and its companion volume, The Content, Impact, and Regulation of Streaming Video. ${ }^{1}$ Both deal with the emerging medium of online video, in its present and in the future. Both share introductions and conclusions.

Technology is the driver. And with digital technology progressing exponentially it is not too early to think about this next generation of television: ever-changing, globalizing, increasingly experiential, individualizing, immersive, often interactive, and absorbing attention away from reality.

When we look ahead, we conclude that the central players in the next generation of television will be companies we call "video cloud platforms" or simply "video clouds." These are operators that provide the intermediary functions of content providers: content aggregation, marketing, customer relations, advertising placement, and billing, together with the technical distribution functions of storage, content delivery, and technological interoperability. They manage complex data operations, and operate next-generation styles of content that are experiential, interactive, and individualized. Increasingly, they also play a role in the production of content itself. We will show why such providers will play a dominant role in the emerging media environment, why there will be relatively few of them in number, and how they will operate globally. They will provide many advantages and opportunities for unprecedented creativity and innovation in technology, content, and business models, but they will also create new problems, or require dealing with old problems in new ways. The companion volume to this book discusses the styles and genres of such TV, the emerging industry impacts of such a market structure, its difficulties, and the policy options for dealing with them.

1 Noam, Eli. The Content, Impact, and Regulation of Streaming Video: The Next Generation of Media Emerges. Cheltenham, UK and Northampton, MA, USA: Edward Elgar Publishing, 2021. 
This new-style television is just emerging, with much dynamism around the world. It thus might seem early to think about its impact and its future. But that is also what people said when broadcast TV started with grainy black-and-white pictures, or when cable "community antenna" cable TV carried four or five channels. We can be certain that a next generation of TV is upon us, and that it will not simply be the same, just more of it.

We are privileged to live in extraordinary times. Within just a few years, we moved from a communication system that provided us, on an individualized basis, with a mere trickle of information (a few kilobits per second of transmission), to a stream (megabits), and soon to a veritable flood of gigabits of ultra-broadband communications. It is the equivalent of stepping up from horses, to trains, to personal automobiles, and to widespread private jets, all within a few years. In the past, we had only very narrow communications pipes available to us individually - telecommunications - and a few bigger pipes that were shared by millions of people watching the same program at the same time: broadcasting. There was only a handful of such "synchronous" broadcast channels. Since then, we have moved to the availability of hundreds of such channels. And we are now transitioning to an infinity of "asynchronous" content streams because it is unnecessary to share usage time with others, except for special live events.

But quantity is only part of the change, and the much easier one to anticipate. The more fundamental change is in the transition of TV from a slow-moving, tightly controlled, standardized technology to one more resembling the dynamics of the internet and information technology (IT). Television has been around since the late 1930s as a consumer medium. In those 80 years, it shifted from an analog black-and-white technology to color digital multicasting at a sharper resolution. By our calculation, its bit rate per distribution channel has increased, if one is generous, by a technological compound annual growth rate (CAGR) of about 4 percent per year. In honor of the guiding spirit of the first decades of mass market TV, this rate should be described as "Sarnoff's Rate," for the leader of the US company RCA which dominated TV technology for its first generation. In contrast, "Moore's Law" rate - with its doubling for every two years - describes technological change in the IT sector based on advances in the underlying semiconductors, and it comes to about 40 percent a year, ten times as fast.

But now TV is migrating to a distribution over the internet. In the process it is moving away from the control of traditional TV organizations. This has been widely noted. But the attention has been mostly on the level of the widening of content options and providers. This is important, of course, but arguably even more fundamental in the long run is the breakdown of the system of substantially uniform TV technology in favor of a system of multiple parallel types of TV. As the video system moves onto the internet and as TV sets become computer-like devices, different technologies can be offered. Competing providers of various technology modules, distribution systems, and content technology will emerge, and their rivalries will transition TV from a system of technical uniformity to one much more resembling that of mobile devices, games, and apps.

As we will discuss, the various technology elements enable TV as a high-resolution, experiential, participatory, individualized, social, mobile, and worldwide experience. Of course, much of the emerging video will continue to be linear, traditional, classic television of a 22-minute program format, surviving in the same way that newspapers, books, and magazines have. But the traditional style will decline in its economic and cultural role. 
To understand the future of media, one must identify the drivers of change, and in what direction they are taking us. This is not engaging in science fiction. We know what the trends are, what technologies can be used for, what leading-edge adopters are already doing, and what technology companies are offering in hardware and applications. Of course, details of developments are unfathomable in advance, but the broad trend is discernable. There is no need to throw up our hands in despair because only part of the scenario of near-future media can be anticipated. When radio emerged in the 1920s, it was so new and different that it was unpredictable. But the same cannot be said for broadcast television and satellite television. For them, one could make strong predictions about where things would be going, based on the experience of the preceding media generation such as radio, plus a thoughtful analysis. More recently, the internet was another paradigm shift whose impact was beyond advance analysis. But once established for text, its application to audio and video was much easier to analyze without resorting to wild guesswork. If there was a problem of analysis, it was often the gold rush mentality permeating the environment, which made rational detached analysis difficult. It has been observed that boosters in general seem to overestimate the receptivity of demand for their new product by a factor of three. In contrast, those who consider adopting a new technology or application tend to err on the side of the familiar and underestimate the benefits to them by a factor of three. ${ }^{2}$ It is this gap between innovators' hopes and buyers' fears that sinks so many promising approaches. These dynamics led, in due course, to the bursting of several hype bubbles, most notoriously after 2000 and 2008, and selectively in subsequent years too. In these bubble situations, we have learned, the regular control institutions did not function well: the journalists, the financial analysts, the accountants - they all succumb to the hype, and to the notion that the old rules do not apply, that the economics of silicon are different, that the economics of bits are different from those of atoms.

It has been characteristic of individuals, institutions, industries, and entire societies to misjudge the future. On the one hand, we tend to succumb to the various merchants of hype, overestimating short-term spread of technology or its salutary impact. On the other hand, we tend to underestimate the long-term impact of fundamental technologies. The automobile and the radio were seen as convenient substitutes to horseless carriages or wireless telegraph, rather than as the agents of revolutions in cityscape and mass media, of living patterns and politics. It is easy, of course, to be smug about the short-sightedness of past generations. But what about our own today? Might we, too, overestimate the short term yet underestimate the long term?

The two books aim to respond to this challenge. Of course, they are not the first on the topic of internet-based media. However, previous volumes often dealt with the entire internet system or with media in general, or they aim to provide skills training for content production. Other books are sociological or cultural and cover the impact of today's social media. A fourth category of books aims to alert the existing media industry of the impending changes. ${ }^{3}$ This book, and its companion volume, aim to absorb these strands and to cast a wide net - from technology to business to content to regulation - while providing a great deal of specifics since

2 Gourville, John T. "Eager Sellers \& Stony Buyers." Harvard Business Review 84, no. 6 (June 2006): 98-106.

3 These books, as well as academic journal articles and other sources, are listed and cited in the various relevant chapters. 
the devil is in the details. In that spirit, this book looks at the next generation of television, with details of the present and a broad perspective on the future. It proceeds as follows:

1. A review of past generations of TV.

2. A review of the new technical elements that are emerging, in both hardware and software.

3. The organizational and technical form of such TV, and the emerging "media clouds."

4. The business models.

5. The fundamental economic characteristics of online $\mathrm{TV}$, and the emerging industry structure.

The companion volume to this book, The Content, Impact, and Regulation of Streaming Video ${ }^{4}$ is centered on content and policy:

1. The technologies of content engineering.

2. How these elements, together, lead to different styles of video content.

3. The societal issues accompanying such next-generation TV.

4. The policy implications of such a video system, and how to deal with several of the problems identified.

5. A way to deal with market power and dominance.

The volumes complement each other but are free-standing. Neither requires the other. Overlaps occur mostly in the Introduction and Outlook chapters, and in the discussion of the factors for media power (Chapter 6 in this volume, Chapter 5 in the companion volume).

The next generation of TV will have a major impact globally, but perhaps nowhere more than on the United States. The US is a major producer of film and TV content to the world (Hollywood). It is also the center of digital technology (Silicon Valley); a center for corporate and start-up financing (Wall Street and the venture capital sector); and of advertising (Madison Avenue). Its defense sector conceived space communications, and many advances in wireless technology, and in packet switching. Its university system set the pace for the internet until recently. And the country's global projection has been based on its "soft power" of its media and culture. ${ }^{5}$ Hence, the US media system is near the forefront of this next wave, for economic, technological, and political reasons. Asian countries have prospered with consumer electronics and are now involved in setting the direction of media technology and business; Europe seeks its role in this next convergence of culture and technology, all while protecting human rights; and many other countries resist the inbound flood of content. There is no place or person in this world that will be left untouched by the impending transformation.

We conclude that a small number of cloud platform providers, which will be vertically integrated into content and data, will dominate the system globally. The reasons, as we will see, are the fundamental economics of such operations. Yet it is equally fundamental that the emerging media system must be wide open to voices and competitors.

Thus, we are at the beginnings of a major change in the video field. The implications for many aspects of society will be deep, wide, and long-lasting. They create a challenge to man-

4 Noam, Eli. The Content, Impact, and Regulation of Streaming Video: The Next Generation of Media Emerges. Cheltenham, UK and Northampton, MA, USA: Edward Elgar Publishing, 2021.

5 The term "culture," as used throughout this book, refers to the practices, arts, science, and social institutions of a society, from their high to their low ends. 
agers, creatives, and policy makers in the digital sector. Otherwise, a backlash will produce political forces that will restrict innovation. It is therefore important for academics, public policy analysts, non-governmental organizations, companies, and governments to think creatively about new approaches to these issues, and to balance the public interest, technological innovation, media diversity, and financial investment in the emerging environment.

These dynamics will be the subject of this book. 